Carlos Machado de Freitas 1 Marcelo Firpo S. Porto 1 Josino Costa Moreira 1 Fatima Pivetta 1 Jorge M. Huet Machado 1 Nilton B. B. de Freitas 2,3 Arline S. Arcuri 4

\section{Segurança química, saúde e ambiente - perspectivas para a governança no contexto brasileiro}

\author{
Chemical safety, health, and environment: \\ prospects for governance in the Brazilian context
}

\footnotetext{
1 Centro de Estudos da Saúde do Trabalhador e Ecologia Humana, Escola Nacional de Saúde Pública, Fundação Oswaldo Cruz. Rua Leopoldo Bulhões 1480, Rio de Janeiro, $R J$ 21041-210, Brasil. cmfreitas@ensp.fiocruz.br 2 Instituto Nacional de Saúde no Trabalho, Central Única dos Trabalhadores. Rua Caetano Pinto 515, São Paulo, SP 03041-000, Brasil. 3 Confederação Nacional dos Químicos, Central Única dos Trabalhadores. Rua Caetano Pinto 515, São Paulo, SP 03041-000, Brasil.

4 Fundação Jorge Duprat Figueiredo de Segurança e Medicina no Trabalho. Rua Capote Valente 710, São Paulo, SP 05409-002, Brasil.
}

\begin{abstract}
Chemical safety is acknowledged by Agenda 21 as one of the most serious problems worldwide, involving governance at the national and international levels. In Brazil, chemical safety problems have increased in intensity and extent, far beyond the capacity to deal with them. The problems are all the more serious in Brazil because issues of democracy, security, sustainability, and equity, all fundamental to governance, are still incipient and still far from being solved. New societal arrangements and a new, contextualized and more participatory science form the basis for developing and expanding strategies for governance to deal with the problem of chemical safety.

Key words Environmental Health; Occupational Health; Chemical Contamination

Resumo A segurança química é reconhecida na Agenda 21 como um dos sérios problemas à saúde e ao ambiente que devem ser enfrentados globalmente, convertendo-se em um problema de governança, nos níveis nacional e internacional. Isto coloca uma série de desafios para países como o Brasil, em que as situações relacionadas a poluição química tiveram um crescimento em intensidade e extensão maiores do que a capacidade de enfrentá-los e controlá-los. Este quadro é ainda mais agravado quando se considera que questões como democracia, segurança, sustentabilidade e equidade, fundamentais para governança, ainda são incipientes e se encontram longe de serem resolvidas. Novos arranjos societários e novas abordagens científicas mais contextualizadas e participativas são as bases para se constituir e expandir estratégias de governança para a segurança química.
\end{abstract}

Palavras-chave Saúde Ambiental; Saúde Ocupacional; Contaminação Química 


\section{Introdução}

A Conferência das Nações Unidas para o Meio Ambiente e Desenvolvimento (CNUMAD), realizada no Rio de Janeiro (Brasil) em 1992, teve como um de seus objetivos o estabelecimento de princípios e compromissos comuns entre as diferentes nações que guiassem um desenvolvimento sustentável da comunidade global, resultando na Agenda 21 (CNUMAD, 1992). Reconhecendo que os países com menor capacidade - institucional e financeira - para enfrentá-los são os mesmos que possuem os maiores desafios em termos de ameaças à sustentabilidade, foi reconhecido, na Agenda 21, que para alcançar os objetivos e as propostas para as ações estabelecidas tornaria-se necessário um significativo fortalecimento dos esforços nacionais e internacionais. Isto incluiria tanto a responsabilidade dos países industrializados em cooperar com os países em industrialização para a solução dos problemas referentes ao meio ambiente e a sustentabilidade, como uma cuidadosa revisão das prioridades e orçamentos visando, progressivamente, internalizar nas economias locais os custos da proteção ao meio ambiente (CGG, 1995; Finkelman, 1996).

É exatamente neste contexto que a segurança química, entendida como um conjunto de estratégias para o controle e a prevenção dos efeitos adversos para o ser humano e o meio ambiente decorrentes da extração, produção, armazenagem, transporte, manuseio e descarte de substâncias químicas, insere-se na Agenda 21. É reconhecido como um dos muitos e sérios problemas essenciais a serem enfrentados globalmente, necessitando-se para tanto ampliar não só a colaboração com os governos, mas também com inúmeros outros atores não governamentais tais como, por exemplo, indústrias, sindicatos, consumidores, organizações não governamentais (ONG), grupos de cidadãos, corporações profissionais e instituições científicas, transformando-se em um problema não só de governabilidade, mais restrita ao papel dos Estados e governos, mas de governança, nos níveis internacional e nacionais.

O Brasil passou, em outubro de 2000, a presidir o Fórum Intergovernamental sobre Segurança Química (FISQ) - seu mandato vai até o ano 2003 - assumindo um papel de liderança internacional no que se refere ao tema. Isto exigirá, mais do que nunca, que os setores saúde, trabalho e meio ambiente, nas suas diversas vertentes de atuação, assim como inúmeros outros setores do governo e atores sociais representando os trabalhadores, os empresários e as comunidades e consumidores expos- tos, sejam capazes de trabalhar de forma integrada para que possamos formular propostas amplas e efetivas para o enfrentamento dos problemas relacionados à poluição química. $\mathrm{O}$ desenvolvimento de metodologias integradas, contextualizadas aos nossos problemas e participativas, assim como processos decisórios mais transparentes e democráticos estão entre os desafios a serem enfrentados.

\section{A segurança química como tema de preocupação internacional}

A segurança química como tema de preocupação internacional aparece já na Conferência Mundial das Nações Unidas sobre Meio Ambiente Humano (CNUMH), realizada em Estocolmo (Suécia), em 1972. As recomendações dessa conferência conduziram ao estabelecimento em 1980 do Programa Internacional de Segurança Química (PISQ), uma joint venture da Organização Mundial da Saúde (OMS), da Organização Internacional do Trabalho (OIT) e do Programa das Nações Unidas para o Meio Ambiente (PNUMA) (Arcuri \& Freitas, 2001; Plestina \& Mercier, 1996). O objetivo inicial do PISQ era prover uma base científica reconhecida internacionalmente, para que os diversos países pudessem desenvolver suas próprias medidas de segurança química (Plestina \& Mercier, 1996).

Vinte anos após a conferência de Estocolmo, foi realizada em 1992, no Brasil, a CNUMAD, que teve como um dos principais documentos aprovados a Agenda 21, onde se encontra o Capítulo 19, exclusivamente dedicado ao tema. Neste capítulo são apontados os problemas de poluição química em grande escala, presentes e futuros, reconhecendo ser a situação mais grave nos países em industrialização por conta da: (1) falta de dados científicos para avaliar os riscos inerentes à utilização de numerosos produtos químicos e; (2) falta de recursos para avaliar os produtos químicos para os quais já se dispõe de dados (CNUMAD, 1992).

Dentre o conjunto de estratégias internacionais fixadas no capítulo 19, foram estabelecidas seis áreas programáticas, que são: (1) expansão e aceleração da avaliação internacional dos riscos químicos; (2) harmonização da classificação e da rotulagem dos produtos químicos; (3) intercâmbio de informações sobre os produtos químicos tóxicos e os riscos químicos; (4) implantação de programas de redução dos riscos; (5) fortalecimento das capacidades e potenciais nacionais para o manejo dos produtos químicos; (6) prevenção do tráfico internacional ilegal dos produtos tóxicos e perigosos. 
Ainda em relação às estratégias internacionais, em 1994 foi criado o FISQ, com o objetivo de constituir um novo mecanismo de cooperação entre governos para promover a avaliação dos riscos das substâncias químicas e sua gestão ecologicamente racional, buscando integrar e unificar os esforços nacionais e internacionais e, ao mesmo tempo, evitar a duplicação de atividades e gastos (IFCS, 1997). Embora se trate de um fórum intergovernamental, é reconhecido que as questões relativas à segurança química, particularmente as referentes às seis áreas programáticas do Capítulo 19, não podem ser levadas a cabo somente pelos governos, tornando-se necessária a participação da indústria, dos diferentes grupos de interesse não governamentais representando comunidades expostas, trabalhadores e organizações intergovernamentais e científicas, entre outros.

Todos estes esforços internacionais referentes à segurança química não podem ser compreendidos de modo descontextualizado. Como é observado pela Comissão sobre Governança Global (CGG, 1995), o crescimento nas quantidades de produtos químicos produzidos tem resultado em níveis de poluição em uma escala tal que vem alterando a composição química das águas, do solo, da atmosfera e dos sistemas biológicos do planeta, colocando em perigo não só o bem-estar, mas também a sobrevivência do planeta.

Especialmente a partir da II Guerra Mundial, o desenvolvimento tecnológico nos processos químicos industriais, impulsionado pela concorrência capitalista e a globalização da economia de escala, vem resultando na expansão da capacidade de produção, armazenamento, circulação e consumo de substâncias químicas em nível mundial. A comercialização de substâncias orgânicas em patamar global é um exemplo disto, passando de sete milhões de toneladas em 1950 para 63 milhões em 1970, 250 milhões em 1985 e mais trezentos milhões no início da década de 90 (Korte \& Coulston, 1994).

Segundo o PISQ, existem mais de 750.000 substâncias conhecidas no meio ambiente, sendo de origem natural ou resultado da atividade humana (IPCS, 1992). Cerca de setenta mil são cotidianamente utilizadas pelo homem, e aproximadamente quarenta mil em significantes quantidades comerciais (IPCS/RPTC, 1992). Desse total, calcula-se que apenas cerca de seis mil substâncias possuam uma avaliação considerada como minimamente adequada sobre os riscos à saúde do homem e ao meio ambiente. Acrescente-se a este quadro a capacidade de inovação tecnológica no ramo químico, que vem colocando disponível no mer- cado, a cada ano, entre mil e duas mil novas substâncias.

Este processo de crescimento do setor químico se encontra estreitamente relacionado ao desenvolvimento de uma economia global altamente interdependente e iníqua, em que a produção, o comércio e os investimentos vêm consolidando um processo de divisão internacional do trabalho, que tem conduzido a uma divisão internacional dos riscos e dos benefícios. Enquanto cerca de $20 \%$ da população mundial, situada principalmente nos países industrializados, consomem aproximadamente $80 \%$ dos bens produzidos, os outros $80 \%$, situados em geral nos países em industrialização, consomem apenas 20\% (MacNeill et al., 1992). $\mathrm{Na}$ Índia, por exemplo, onde houve o acidente químico ampliado mais grave registrado em toda a história da humanidade (mais de 2.500 óbitos imediatos na cidade de Bhopal, em 1984), o consumo de produtos resultantes da tecnologia química era de $1 \mathrm{~kg}$ per capita, enquanto nos países industrializados esse consumo era de 30 a 40kg per capita (Murti, 1991).

Diante da complexidade e amplitude dos problemas provenientes da poluição química ambiental, que vem desafiando cada vez mais a capacidade dos governos no que tange à segurança e à saúde dos cidadãos, notadamente nos países industrializados, a segurança química converte-se em uma das questões globais de governança. Expressa a constatação de que o nosso futuro comum depende não somente do crescimento econômico, mas também da melhoria da qualidade de vida, sobremodo para as populações mais pobres, tendo por base os princípios de universalidade, solidariedade e eqüidade, que devem ser mantidos, além de orientar as decisões e ações sobre segurança química nos níveis global e local (CGG, 1995; Filkenman, 1996).

\section{Segurança química e governança no contexto da complexidade e da vulnerabilidade}

Conforme é afirmado no relatório da CGG (1995), a mobilização do poder coletivo das pessoas para tornar a vida no século XXI mais democrática, mais segura, mais sustentável e com eqüidade é o grande desafio de nossa geração. Isto implica a necessidade das nações e da comunidade mundial assumirem a grande responsabilidade coletiva que lhes é colocada em relação a estas questões, que se encontram intrinsecamente relacionadas e em que a segurança deixa de ser a dos Estados, mas passa a 
ser prioritariamente a das pessoas. A segurança química, entendida como um dos tantos e importantes aspectos relativos à segurança da saúde, da vida e da proteção ao meio ambiente, em virtude das ameaças presentes e futuras, coloca-se neste contexto como uma questão de governança, nos níveis global e local, não se restringindo aos governos e às inter-relações governamentais. É um desafio ainda maior em países como o Brasil, em que as questões concernentes à democracia, à segurança, à sustentabilidade e à eqüidade, fundamentais para a governança, se encontram ainda pouco resolvidas e incipientes e, por isso, devem ser integradas à questão da segurança química no País.

A construção de políticas de segurança química nos países em industrialização como o Brasil, além de levar em consideração o enfrentamento da complexidade e das incertezas referentes à compreensão do problema, as quais se ampliam por conta da diversidade e da precariedade dos mesmos, deve considerar também os aspectos referentes aos diferentes modos e níveis de vulnerabilidades, na busca de construção de conhecimentos e processos decisórios mais contextualizados e participativos, nos níveis local e global, como pré-requisitos básicos para a governança.

A noção de complexidade aplicada aos problemas relacionados à poluição química implica que não podemos reduzir suas análises a componentes isolados, como operado pelas abordagens tradicionais da ciência, uma vez que implicaria tanto perdas importantes na compreensão dos problemas, ampliando as incertezas, como na limitação na formulação de estratégias de prevenção e controle de tais riscos (Funtowicz \& Ravetz, 1993). Em relação a isto, Funtowicz \& Ravetz (1993) distinguem três níveis de incertezas. As incertezas técnicas, relacionadas à inexatidão dos dados e das análises, e que podem ser gerenciadas por meio de rotinas padronizadas adequadas desenvolvidas por campos científicos particulares. As incertezas metodológicas, relacionadas à não-confiabilidade dos dados e que envolvem aspectos mais complexos e relevantes da informação, como valores e confiabilidade. Finalmente as incertezas epistemológicas, relacionadas às margens de ignorância do próprio conhecimento científico, sendo este nível envolvido quando irremediáveis incertezas encontram-se no centro do problema (Funtowicz \& Ravetz, 1993).

Enfrentar as incertezas inerentes ao nosso atual modo científico de avaliar os problemas de origem química e compreender o problema de modo amplo e sistêmico envolve, então, integrar os múltiplos e simultâneos aspectos de diferentes naturezas. Nesta perspectiva, as políticas globais e locais de produção, transporte, comercialização, armazenamento, descarte e segurança, assim como as direções dadas ao desenvolvimento da tecnologia química estarão, simultaneamente e de modo inextricável, interagindo com as emissões de substâncias químicas que atingirão solos, águas, atmosfera e cadeia alimentar. Estas emissões, mediadas por reações químicas e relações sociais, culturais, econômicas e de poder, resultarão tanto nos diferentes níveis de contaminação dos seres humanos e ecossistemas, como nos diferentes níveis de capacidade de resposta social ao problema. Isto implica que os processos decisórios sobre riscos químicos com vistas à governança não podem ser realizados tendo-se por base somente as limitadas predições técnico-científicas, exigindo-se que considerações acerca dos inúmeros aspectos apontados, assim como, também, os inerentes valores e interesses em jogo, façam parte dos mesmos, complementado os aspectos de políticas públicas (Funtowicz \& Ravetz, 1993). Como observam De Marchi \& Ravetz (1999), muitos dos novos riscos, como os de origem química, combinam extremas incertezas com possibilidade de danos extensivos e irreversíveis, exigindo novas formas de processos decisórios.

Para que novas abordagens e novos processos decisórios sejam minimamente viáveis, particularmente no contexto dos países em industrialização, devemos considerar o conceito de vulnerabilidade (Horlick-Jones, 1993). Para nós, a vulnerabilidade deve ser subdividida em duas, que se inter-relacionam. A primeira refere-se à vulnerabilidade populacional (Morrow, 1999) e relaciona-se à existência de grupos populacionais vulneráveis, de acordo com suas características em termos de status social, político e econômico, etnicidade, gênero, incapacidade, idade etc., sendo isto derivado de variadas formas e níveis de exclusão social. A segunda refere-se à vulnerabilidade institucional (Barrenechea, 1998) e relaciona-se ao funcionamento da sociedade em termos das políticas públicas, processos decisórios e das instituições que atuam nos condicionantes estruturais ou pressões dinâmicas que propiciam ou agravam as situações e eventos de riscos. No Brasil, consideramos que muito ainda deve ser feito, a fim de que a segurança química na sua interface com a governança possa ser realizada, especialmente quando se considera que no atual contexto o Estado vem sendo continuamente desestruturado, tornando-se incapaz de controlar e prevenir os problemas de origem química, caracterizando uma vulnerabilidade ins- 
titucional. Este quadro é agravado pelo fato de determinados grupos sociais estarem sendo expostos a substâncias químicas em situações sociais e ambientais precárias, caracterizando uma vulnerabilidade populacional.

Nesta perspectiva, a governança só poderá florescer se fundada em um forte compromisso com os princípios de eqüidade e democracia baseados na sociedade civil. Os princípios da governança se encontram em consonância com as perspectivas da saúde coletiva, devendo orientar as decisões e ações para o setor saúde no Brasil, particularmente quando envolvem questões ambientais, em que o leque de atores e interesses heterogêneos se amplia.

\section{Segurança química e governança na realidade brasileira}

O Brasil, dentre outros países em industrialização, como Índia e México, sofreu um processo de intensificação de seu crescimento econômico entre os anos 60 e 80 mediante grande endividamento externo - se encontra entre os países com maior dívida externa -, aumento da participação de indústrias multinacionais no processo de industrialização e forte intervenção do Estado na economia. Em 1990, dentre os segmentos constituintes do setor industrial, o químico representava cerca de $19 \%$ da produção do país. De acordo com a Pesquisa Industrial Anual do IBGE (Fundação Instituto Brasileiro de Geografia e Estatística), ano de 1997, do total da receita líquida de vendas de todo o setor industrial brasileiro, a indústria química respondeu por cerca de $22 \%$ (IBGE, 1997) e na atualidade ocupa um lugar de destaque no mundo, encontrando-se em oitavo lugar.

No Brasil, o modelo de desenvolvimento econômico adotado, sustentado pela ausência de um sistema político democrático - particularmente entre os anos 60 e 80 - e grandes transformações na sociedade, combinando concentração de capital, exploração da mãode-obra e abandono ou omissão do poder público no controle e prevenção dos riscos químicos, resultou em rápida e desordenada industrialização. Paralelamente, ocorreu um intenso e incontrolado processo de urbanização, acompanhado de grande fluxo migratório do campo e das regiões mais pobres para os grandes centros urbanos, relegando ao plano secundário os problemas sociais, humanos ou ambientais (Becker \& Egler, 1993). Uma das conseqüências desse processo foi o assentamento de parte dessas populações pobres e com baixo nível de escolaridade, que migraram do campo na busca de melhores condições de vida e trabalho, nas áreas periféricas dos grandes centros urbanos, passando a viver em condições precárias e sem acesso aos bens e serviços básicos de saneamento, saúde e educação. Situação similar, em termos de condições precárias de vida e trabalho, ocorreu também para aqueles que ficaram nas áreas rurais. O resultado foi a constituição de padrões inferiores de segurança e de proteção ambiental e à saúde não só no nível internacional, mas também no nível interno dos países de economia periférica, definindo, assim, as áreas salubres e seguras e as insalubres e inseguras (Barbosa, 1992; Guilherme, 1987; Torres, 1993).

Nas áreas rurais, são bem conhecidos os casos de contaminação por agrotóxicos de trabalhadores e de suas famílias, bem como moradores das áreas próximas expostos à contaminação ambiental (águas, ar e solos) e da cadeia alimentar, num circuito de complexas interações químicas e sociais. Tendo origem também nos problemas estruturais resultantes dos modelos de desenvolvimento adotados no país, a ausência de uma política de reforma agrária e de oferta de trabalhos estáveis contribuiu para fluxos migratórios do campo não só para as cidades, mas também para áreas de mineração, caso dos garimpos de ouro na região amazônica. Se, por um lado, as atividades de mineração do ouro fornecem a maior taxa de empregos da região $(10,7 \%)$, coexistindo com precárias condições sanitárias e um quadro de doenças endêmicas como a malária e a leishmaniose, por outro, tais atividades vêm resultando na intensa degradação do meio ambiente e profunda desorganização e exclusão social (MMA, 1995). Freqüentemente, trata-se de atividade ilegal que envolve força de trabalho precarizada, não qualificada, móvel e sem direitos trabalhistas - algumas vezes envolvendo até o trabalho escravo -, que se organiza em núcleos, em torno das minerações, e estabelecem interfaces entre as formas de exploração mecanizadas das empresas e as manuais dos garimpeiros. Pelo fato de, em grande parte, as técnicas adotadas serem rudimentares, acabam por empregar grandes quantidades de mercúrio $(\mathrm{Hg})$, resultando em elevados níveis de poluição do ar, sedimentos e águas dos rios, contaminando os trabalhadores garimpeiros e de casas de queima. Além destes trabalhadores, populações urbanas que vivem próximas a casas de queima e aos garimpos, bem como as ribeirinhas, acabam, por interações ambientais e vias diretas ou indiretas, sendo contaminadas com metilmercúrio (Câmara \& Corey, 1993; MMA, 1995). 
A complexa trama social que envolve a atividade de mineração do ouro se conjuga com a complexidade ambiental associada à capacidade de biotransformação do Hg para sua forma mais tóxica - o metilmercúrio. Isto é agravado pelas incertezas oriundas tanto da ausência de dados científicos sobre o seu comportamento em ambientes tropicais, bem como as relacionadas aos problemas que poderão ocorrer com o ciclo do mesmo devido às mudanças climáticas globais que poderão contribuir para enriquecer a remobilização e bioacumulação deste agente químico. $\mathrm{O}$ resultado será o aumento do risco de exposição pela exalação deste agente, convertendo-se em bombas químicas de tempo (Nriagu, 1999).

Nos grandes centros urbanos os problemas de origem química se manifestam de diversas formas, indo da produção em pequenas indústrias - como no caso das fábricas ou reformadoras de baterias - e grandes indústrias do setor químico - envolvendo as químicas, petroquímicas e petroleiras -, até o destino final dos resíduos químicos. Um dos mais conhecidos e paradigmáticos casos de contaminação ambiental por resíduos perigosos envolvendo a combinação de vulnerabilidade institucional e populacional é o da Cidade dos Meninos, no Município de Duque de Caxias, Rio de Janeiro. Neste local houve, em 1954, o fechamento de uma fábrica do Ministério da Saúde (MS), ocorrendo o abandono de cerca de setecentas toneladas de resíduos da produção de $\mathrm{HCH}$ (grau técnico) utilizados em campanhas contra a malária. Esta área é hoje habitada por cerca de 1.500 pessoas e os resíduos foram encontrados em todos os segmentos ambientais, nos habitantes e biota locais em níveis extremamente elevados (Oliveira et al., 1995).

Outro sério problema se refere às pequenas fábricas que empregam substâncias químicas, muitas de fundo de quintal. Reformadoras de baterias, por exemplo, se localizam, em sua quase totalidade, em áreas residenciais e comerciais em que vivem populações de baixa renda. Estas indústrias empregam, em geral, perto de dez trabalhadores, caracterizados por possuírem baixa escolaridade e nenhum tipo de treinamento e informações quanto aos riscos e às atitudes de proteção e segurança que deveriam ser tomadas. Suas instalações são inadequadas e utilizam um processo de trabalho simples e arcaico, provocando a contaminação por chumbo não apenas dos trabalhadores, mas atingindo também as áreas em torno e populações circunvizinhas (Silva \& Mattos, 1999). Funcionam com elevados custos marginais, ficando ao largo dos programas de incen- tivos econômicos relacionados a melhorias de desempenho ambiental; raramente são alvos de fiscalização pelos órgãos públicos que, no atual quadro, se atuassem efetivamente, poderiam acirrar a crise social pelo possível fechamento das mesmas, resultando no aumento do número de desempregados.

Diferentemente dos exemplos anteriores, os casos de poluição crônica e acidentes nas grandes indústrias têm, freqüentemente, envolvido os trabalhadores da indústria química, que possuem alto nível de qualificação técnica, educação formal e maior capacidade de organização local - pelos sindicatos - e nacional e, por conseguinte, maior poder de mobilizações e pressões sociais. Apesar dos eventuais limites desta participação, algumas experiências ocorridas na década de 90 , como a participação destes trabalhadores em acordos e comissões nacionais que envolveram e vêm envolvendo, de maneira conjunta, representantes das indústrias e do governo, como nos casos do benzeno, da norma sobre vasos e caldeiras, e ainda sobre a construção de uma legislação nacional sobre os acidentes industriais ampliados demonstraram a possibilidade de se produzirem processos decisórios mais democráticos e na perspectiva da governança.

Tendo como referência os casos citados, podemos considerar que houve no Brasil um crescimento dos problemas relacionados à segurança química em uma intensidade e amplitude maior do que a capacidade do Brasil enfrentá-los. Neste contexto, a reconhecida complexidade sócio-ambiental do Brasil associada às vulnerabilidades populacional e institucional, vem, por décadas seguidas, propiciando a utilização indiscriminada dos recursos naturais e sua contaminação pela coexistência de modos de produção arcaicos com os de tecnologia avançada, resultando em diferentes formas e níveis de inserção social e poluição química.

Hoje, a gestão da segurança química no Brasil pelos governos federal, estadual ou municipal, vem sendo desenvolvida de maneira ineficiente e com pouca integração entre os vários setores e grupos sociais envolvidos, tendo como conseqüências o conflito de competências entre diferentes órgãos dos governos, omissões e a falta de capacidade instalada de recursos humanos e técnicos, mormente no que se refere à proteção da saúde e do meio ambiente. Embora o arcabouço legal disponível possa ser considerado relativamente vasto, na prática não se mostra factível frente à contínua desestruturação dos órgãos de governo, sendo isto em parte o resultado das descontinuidades das 
políticas públicas e da falta de recursos financeiros para os setores ambiental e de saúde.

No atual contexto, políticas auto-regulatórias, como a certificação pela norma ISO 14.000 ou programas voluntários como o Atuação Responsável da indústria química, correm o risco de substituir de forma inapropriada a carência de políticas públicas. Ainda mais quando se considera que, com a relativa estagnação econômica das últimas duas décadas, aliada ao desemprego estrutural inerente ao modelo de desenvolvimento econômico atualmente em vigor, a exclusão social vem se acentuando e reduzindo o poder de pressão da sociedade, mesmo dos trabalhadores industriais, um importante grupo social de pressão na questão da $s e$ gurança química e que tiveram papel ativo e fundamental em alguns exemplos apresentados, os quais minimamente atendiam aos princípios para a governança.

\section{Conclusão}

O Estado no Brasil, tanto quanto em outros países em industrialização, tem caminhado em um sério e perigoso processo de deterioração, com crescente alienação e indiferença às necessidades e demandas da população. Em um contexto como este, Finkelman (1996) observa que torna-se premente a redefinição do papel do Estado em cada um dos níveis e ações que concernem à segurança química, em particular nas áreas que possuem responsabilidades mais diretas em relação ao tema, como as instituições que provêem diretamente atenção à saú- de das populações e as que são responsáveis pelo controle e a proteção do meio ambiente.

A segurança química, como um sério problema a ser enfrentado por países como o Brasil, coloca a necessidade e o desafio de se constituírem novos arranjos societários nos níveis global, regional, nacional e local em busca de um modelo de desenvolvimento sustentável que tenha por base a eqüidade e a democracia. Outro desafio é se constituir, concomitantemente, uma ciência mais contextualizada na nossa realidade, baseada em abordagens integradas e participativas que possam incluir a análise de reações químicas, físicas e biológicas combinadas com reações sociais, políticas, culturais, éticas e morais, contribuindo para a busca de soluções mais amplas e duradouras.

A segurança química não é um tema descontextualizado do mundo atual, em que a maioria da população do planeta vive excluída dos benefícios da modernização/globalização. Esta mesma população, no papel que lhe cabe na divisão internacional do trabalho, vem arcando com os riscos de um modelo de desenvolvimento iníquo em sua natureza e dinâmica. Poucas tentativas têm sido realizadas em anos recentes para retificar tal situação. Ainda que possa se considerar que muitos indicadores de progresso social - mortalidade infantil, educação, expectativa de vida e nutrição - melhoraram significativamente em termos de médias globais, milhões de pessoas no planeta, expostos à poluição química, ainda vivem a ausência de água potável e saneamento (CGG, 1995).

\section{Referências}

ARCURI, A. S. A. \& FREITAS, N. B. B., 2001. Anais do Seminário Internacional de Segurança Química o Contexto Brasileiro. São Paulo: Fundação Jorge Duprat Figueiredo de Segurança e Medicina no Trabalho.

BARBOSA, S. R. C. S., 1992. Ambiente, qualidade de vida e cidadania. Algumas reflexões sobre regiões urbano-industriais. In: Dilemas Socioambientais e Desenvolvimento Sustentável (D. J. Hogan \& P. F. Vieira, org.), pp. 193-210, Campinas: Universidade Estadual de Campinas. 
BARRENECHEA, J., 1998. Estrategias Político Institucionales de Riesgo Tecnológico Industrial. El Caso de Zárate y Camapana, Provincia de Buenos Aires. Buenos Aires: Universidad de Buenos Aires.

BECKER, B. K. \& EGLER, C., 1993. Brasil: Uma Nova Potência Regional na Economia do Mundo. São Paulo: Bertrand.

CÂMARA, V. \& COREY, G., 1993. O Casos dos Garimpos de Ouro no Brasil - Epidemiologia e Meio Ambiente. Metepec: Centro Pan-Americano de Ecologia Humana e Saúde/Organização Pan-Americana de Saúde.

CGG (Commission on Global Governance), 1995. An Overview of Our Global Neighborhood - The Report of the Commission on Global Governance. London: United Nations.

CNUMAD (Conferência das Nações Unidas sobre Meio Ambiente e Desenvolvimento), 1992. Conferência das Nações Unidas sobre Meio Ambiente e Desenvolvimento - Agenda 21. Brasília: Senado Federal.

DE MARCHI, B. \& RAVETZ, J., 1999. Risk management and governance: A post-normal science approach. Futures, 31:743-757.

FINKELMAN, J., 1996. Chemical safety and health in Latin America: An overview. Science of the Total Environment, 188(Sup. 1):S3-S29.

FUNTOWICZ, S. \& RAVETZ, J., 1993. Science for the post-normal age. Futures, 25:739-755.

GUILHERME, M. L., 1987. Urbanização, saúde e meio ambiente - O caso da implantação do Pólo Industrial de Cubatão e seus efeitos urbanos e regionais nos setores saúde e poluição ambiental. Espaço e Debates, 22:42-53.

HORLICK-JONES, T., 1993. Patterns of risk and patterns of vulnerability. In: Workshop on Emergency Management (A. Amendola \& B. de Marchi, ed.), pp. 33-42, Rome: Institute for Systems Engineering and Informatics, Joint Research Centre, Commission of the European Communities.

IFCS (International Forum on Chemical Safety), 1997. Executive Summary - Forum II Agreed Action Items and Recommendations. Ottawa: IFCS.

IPCS (International Programme on Chemical Safety), 1992. IPCS News - The Newsletter of the International Programme on Chemical Safety, 01.

IPCS (International Programme on Chemical Safety)/IRPTC (International Register of Potentially Toxic Chemicals), 1992. Computadorized Listining of Potentially Toxic Chemicals. Geneva: World Health Organization/United Nations Environment Program/International Labour Organization.
IBGE (Fundação Instituto Brasileiro de Geografia e Estatística), 1997. Anuário Estatístico do Brasil. v. 57. Rio de Janeiro: IBGE.

KORTE, F. \& COULSTON, F., 1994. Some considerations of the impact of energy and chemicals on the environment. Regulatory Toxicology and Pharmacology, 19:219-227.

MacNEILL, J.; WINSEMIUS, P. \& YAKUSHIJI, T., 1992. Para Além da Interdependência - A Relação Entre a Economia Mundial e a Ecologia da Terra. Rio de Janeiro: Jorge Zahar Editor.

MMA (Ministério do Meio Ambiente, dos Recursos Hídricos e da Amazônia Legal), 1995. Os Ecossistemas Brasileiros e os Principais Macrovetores de Desenvolvimento - Subsídios ao Planejamento da Gestão Ambiental. Projeto Cenário para o Planejamento da Gestão Ambiental. Brasília: MMA.

MORROW, B. H., 1999. Identifying and mapping community vulnerability. Disaster, 23:1-18.

MURTI, C. R. K., 1991. Industrialization and emerging environmental health issues: Lessons from the Bophal disaster. Toxicology and Industrial Health, 7:153-164.

NRIAGU, J. O., 1993. Mercury cycle and global climate change. In: $5^{\text {th }}$ International Conference "Mercury as Global Pollutant", Proceedings, p. 1. Rio de Janeiro: Centro de Tecnologia Mineral.

OLIVEIRA, R. M.; BRILHANTE, O. M.; MOREIRA, J. C. \& MIRANDA, A. C., 1995. Contaminação por hexaclorociclohexanos em área urbana da Região Sudeste do Brasil. Revista de Saúde Publica, 29: 228-233.

PLESTINA, R. \& MERCIER, M., 1996. Human health and environmental hazards arising from the use of chemicals in developing countries. Chemical safety and health in Latin America: An overview. Science of the Total Environment, 188(Sup. 1): S135-S140.

TORRES, H., 1993. A Emergência das Indústrias “Sujas" e Intensivas em Recursos Naturais no Cenário Industrial Brasileiro. Documento de Trabalho 9. Brasília: Instituto Sociedade, População e Natureza.

SILVA, C. R. S. \& MATTOS, R. C. O. C., 1999. Avaliação da Exposição ao Chumbo de Trabalhadores de Fábricas e Reformadoras de Baterias e das Populações Circunvizinhas no Rio de Janeiro. Relatório Técnico. Rio de Janeiro: Centro de Estudos da Saúde do Trabalhador e Ecologia Humana, Escola Nacional de Saúde Pública, Fundação Oswaldo Cruz.

Recebido em 18 de janeiro de 2001

Versão final reapresentada em 31 de junho de 2001

Aprovado em 1 de agosto de 2001 